SQUARE-FULL DIVISORS OF SQUARE-FULL INTEGERS

\author{
A. H. Ledoan \\ Dept. of Mathematics, University of Rochester, Rochester, New York \\ ledoan@math.rochester.edu \\ A. Zaharescu ${ }^{1}$ \\ Dept. of Mathematics, University of Illinois at Urbana-Champaign, Urbana, Illinois \\ zaharesc@math.uiuc.edu
}

Received: 8/17/09, Accepted: 2/11/10, Published: 5/24/10

\begin{abstract}
We consider some general real moments associated with square-full divisors of square-full numbers and compute the contribution to these moments given by the square divisors.
\end{abstract}

\title{
1. Introduction and Statement of Results
}

A positive integer $n$ is called square-full if, in the canonical representation of $n$ into prime powers, each exponent is at least 2. Let $L$ denote the set of square-full integers, and define $l$ by $l(n)=1$ or $l(n)=0$ according to whether $n \in L$ or $n \notin L$. Let $L(x)$ denote the enumerative function of the set $L$,

$$
L(x)=\sum_{n \leq x} l(n),
$$

where $x$ is a real variable $\geq 1$.

In 1934, Erdős and Szekeres [4] proved the asymptotic formula

$$
L(x)=\frac{\zeta(3 / 2)}{\zeta(3)} x^{1 / 2}+O\left(x^{1 / 3}\right),
$$

where $\zeta(s)$ denotes the Riemann zeta-function. A simple proof of this result was later given by Sklar [5]. In 1954, Bateman [1] improved this result by means of the Euler-MacLaurin summation formula to

$$
L(x)=\frac{\zeta(3 / 2)}{\zeta(3)} x^{1 / 2}+\frac{\zeta(2 / 3)}{\zeta(2)} x^{1 / 3}+O\left(x^{1 / 5}\right) .
$$

Bateman remarked that, by more delicate methods, it is possible to sharpen the error term above to $O\left(x^{1 / 6}(\log x)^{2}\right)$.

\footnotetext{
${ }^{1}$ Supported in part by NSF grant number DMS-0901621.
} 
In the present paper, we study some statistical properties of the square-full divisors of square-full integers. The probability that a randomly selected square-full integer in the interval $[1, x]$ is a square approaches $\zeta(3) / \zeta(3 / 2)$ as $x$ tends to infinity. What is the probability that a randomly selected square-full divisor of a randomly chosen square-full number $\leq x$ is a square? More precisely, does the ratio

$$
\frac{\sum_{\substack{n \leq x \\ \text { square-full }}} \#\{1 \leq d \leq n ; d \mid n, d \text { is a square }\}}{\sum_{\substack{n \leq x \\ n \text { square-full }}} \#\{1 \leq d \leq n ; d \mid n, d \text { is square-full }\}}
$$

have a limit as $x$ tends to infinity? This type of question leads us to consider some general real moments associated with square-full divisors of square-full numbers, and to compute the contribution to these moments given by the square divisors.

Fix two positive real numbers $\alpha$ and $\beta$. For each $n$, we consider the divisor sums

$$
\sigma_{\text {square-full }, \alpha, \beta}(n)=\sum_{\substack{d_{1} d_{2}=n \\ d_{1}, d_{2} \\ \text { square-full }}} d_{1}^{\alpha} d_{2}^{\beta}
$$

and

$$
\sigma_{\text {square }, \alpha, \beta}(n)=\sum_{\substack{d_{1} d_{2}=n \\ d_{1} \text { square, } d_{2} \text { square-full }}} d_{1}^{\alpha} d_{2}^{\beta} .
$$

Here we note that $\sigma_{\text {square-full }, \alpha, \beta}(n)=\sigma_{\text {square }, \alpha, \beta}(n)=0$, unless $n$ is square-full. Also, for $n$ square-full and both $\alpha=0$ and $\beta=0$, we see that $\sigma_{\text {square-full, } \alpha, \beta}(n)$ counts the number of square-full divisors $d$ of $n$ with the property that $n / d$ is square-full, while $\sigma_{\text {square-full, } \alpha, \beta}(n)$ counts the number of square divisors $d$ of $n$ with the property that $n / d$ is square-full. We consider the weighted moments

$$
M_{\text {square-full }, \alpha, \beta}(x)=\sum_{\substack{n \leq x \\ n \text { square-full }}}(1-n / x) \sigma_{\text {square-full, } \alpha, \beta}(n)
$$

and

$$
M_{\text {square }, \alpha, \beta}(x)=\sum_{\substack{n \leq x \\ n \text { square-full }}}(1-n / x) \sigma_{\text {square }, \alpha, \beta}(n) .
$$

We wish to see whether the limit

$$
\lim _{x \rightarrow \infty} \frac{M_{\text {square }, \alpha, \beta}(x)}{M_{\text {square-full, } \alpha, \beta}(x)}
$$


exists. Further, we are interested to see for which values of $\alpha$ and $\beta$, if any, this limit equals the proportion of squares in the sequence of square-full numbers, which is $\zeta(3) / \zeta(3 / 2)$. We will prove the following asymptotic formulas.

Theorem 1 Fix two real numbers $\alpha, \beta \geq 0$. As $x \rightarrow \infty$,

$$
M_{\text {square-full, } \alpha, \beta}(x) \sim \begin{cases}\frac{\zeta(2 \alpha-2 \beta+1) \zeta(3 \alpha-3 \beta+3 / 2) \zeta(3 / 2)}{2(\alpha+1 / 2)(\alpha+3 / 2) \zeta(6 \alpha-6 \beta+3) \zeta(3)} x^{\alpha+1 / 2}, & \text { if } \alpha>\beta, \\ \frac{\zeta^{2}(3 / 2)}{\left(4 \alpha^{2}+8 \alpha+3\right) \zeta^{2}(3)} x^{\alpha+1 / 2} \log x, & \text { if } \alpha=\beta, \\ \frac{\zeta(2 \beta-2 \alpha+1) \zeta(3 \beta-3 \alpha+3 / 2) \zeta(3 / 2)}{2(\beta+1 / 2)(\beta+3 / 2) \zeta(6 \beta-6 \alpha+3) \zeta(3)} x^{\beta+1 / 2}, & \text { if } \alpha<\beta,\end{cases}
$$

and

$$
M_{\text {square }, \alpha, \beta}(x) \sim \begin{cases}\frac{\zeta(2 \alpha-2 \beta+1) \zeta(3 / 2)}{2(\alpha+1 / 2)(\alpha+3 / 2) \zeta(3)} x^{\alpha+1 / 2}, & \text { if } \alpha>\beta, \\ \frac{\zeta(3 / 2)}{\left(4 \alpha^{2}+8 \alpha+3\right) \zeta(3)} x^{\alpha+1 / 2} \log x, & \text { if } \alpha=\beta, \\ \frac{\zeta(2 \beta-2 \alpha+1) \zeta(3 / 2)}{2(\beta+1 / 2)(\beta+3 / 2) \zeta(3)} x^{\beta+1 / 2}, & \text { if } \alpha<\beta .\end{cases}
$$

More accurate asymptotic formulas will be provided in the last section. As a consequence of Theorem 1, we have the following corollary.

Corollary 2 Fix two real numbers $\alpha, \beta \geq 0$. Then

$$
\lim _{x \rightarrow \infty} \frac{M_{\text {square }, \alpha, \beta}(x)}{M_{\text {square-full }, \alpha, \beta}(x)}= \begin{cases}\frac{\zeta(6 \alpha-6 \beta+3)}{\zeta(3 \alpha-3 \beta+3 / 2)}, & \text { if } \alpha>\beta, \\ \frac{\zeta(3)}{\zeta(3 / 2)}, & \text { if } \alpha=\beta, \\ \frac{\zeta(6 \beta-6 \alpha+3)}{\zeta(3 \beta-3 \alpha+3 / 2)}, & \text { if } \alpha<\beta .\end{cases}
$$

It is easy to see from the associated Euler product that

$$
\frac{\zeta(6 \alpha-6 \beta+3)}{\zeta(3 \alpha-3 \beta+3 / 2)}>\frac{\zeta(3)}{\zeta(3 / 2)}
$$

for $\alpha>\beta$, and

$$
\frac{\zeta(6 \beta-6 \alpha+3)}{\zeta(3 \beta-3 \alpha+3 / 2)}>\frac{\zeta(3)}{\zeta(3 / 2)}
$$

for $\alpha<\beta$. Therefore, by Corollary 2 ,

$$
\lim _{x \rightarrow \infty} \frac{M_{\text {square }, \alpha, \beta}(x)}{M_{\text {square-full }, \alpha, \beta}(x)} \geq \frac{\zeta(3)}{\zeta(3 / 2)}
$$

for all $\alpha, \beta \geq 0$, with equality exactly when $\alpha=\beta$. 


\section{Proof of Theorem 1}

In this section, we give a detailed proof for the first part of Theorem 1, and we indicate the modifications needed for the second part.

Proof. To commence, let $s$ be a complex variable and consider the Dirichlet series

$$
F_{\alpha, \beta}(s)=\sum_{n=1}^{\infty} \frac{\sigma_{\text {square-full }, \alpha, \beta}(n)}{n^{s}} .
$$

Here we note that for any $n$,

$$
\begin{aligned}
\sigma_{\text {square-full }, \alpha, \beta}(n) & \leq n^{\max (\alpha, \beta)} \#\left\{d_{1} d_{2}=k ; d_{1}, d_{2} \text { square-full }\right\} \\
& \ll n^{\max (\alpha, \beta)+O(1 / \log \log n)} .
\end{aligned}
$$

(See, for example, Ramanujan [3] or Hardy and Wright [2].) Combining this with the fact that $\sigma_{\text {square-full }, \alpha, \beta}(n)=0$ unless $n$ is square-full, it follows that the Dirichlet series (1) converges in the region $\Re s>1 / 2+\max (\alpha, \beta)$.

By Perron's inversion formula (see Titchmarsh [6], Section 9.42, pp. 300-301; or Titchmarsh [7], Section 3.12, pp. 60-62), we have

$$
\begin{aligned}
M_{\text {square-full }, \alpha, \beta}(x) & =\sum_{n \leq x}(1-n / x) \sigma_{\text {square-full }, \alpha, \beta}(n) \\
& =\frac{1}{2 \pi i} \int_{c-i \infty}^{c+i \infty} \frac{x^{s}}{s(s+1)} F_{\alpha, \beta}(s) d s,
\end{aligned}
$$

for any $c>1 / 2+\max (\alpha, \beta)$. We now rewrite $F_{\alpha, \beta}(s)$ as

$$
\begin{aligned}
F_{\alpha, \beta}(s) & =\sum_{n=1}^{\infty} \frac{1}{n^{s}} \sum_{\substack{d_{1} d_{2}=n \\
d_{1}, d_{2} \\
\text { square-full }}} d_{1}^{\alpha} d_{2}^{\beta} \\
& =\sum_{\substack{d_{1}=1 \\
d_{1}}}^{\infty} \sum_{\substack{d_{2}=1 \\
\text { square-full } \\
d_{2}}}^{\infty} \frac{d_{1}^{\alpha} d_{2}^{\beta}}{\left(d_{1} d_{2}\right)^{s}} \\
& =\sum_{\substack{d_{1}=1 \\
\text { square-full }}}^{\infty} \frac{1}{d_{1}^{s-\alpha}} \sum_{\substack{d_{1}=1 \\
\text { square-full }}}^{\infty} \frac{1}{d_{2}^{s-\beta}} .
\end{aligned}
$$

Consider the Dirichlet series

$$
H(s)=\sum_{m \text { square-full }} \frac{1}{m^{s}},
$$


which converges for $\Re s>1 / 2$. Then

$$
F_{\alpha, \beta}(s)=H(s-\alpha) H(s-\beta),
$$

and substituting this into (2), we obtain

$$
M_{\text {square-full }, \alpha, \beta}(x)=\frac{1}{2 \pi i} \int_{c-i \infty}^{c+i \infty} \frac{x^{s}}{s(s+1)} H(s-\alpha) H(s-\beta) d s .
$$

Expressing the function $H(s)$ in terms of $\zeta(s)$, we find that

$$
\begin{aligned}
H(s) & =\prod_{p \text { prime }}\left(1+\frac{1}{p^{2 s}}+\frac{1}{p^{3 s}}+\frac{1}{p^{4 s}}+\ldots\right) \\
& =\prod_{p \text { prime }}\left(1+\frac{1}{p^{2 s}}+\frac{1}{p^{4 s}}+\ldots\right)\left(1+\frac{1}{p^{3 s}}\right) \\
& =\prod_{p \text { prime }} \frac{1-1 / p^{6 s}}{\left(1-1 / p^{2 s}\right)\left(1-1 / p^{3 s}\right)} \\
& =\frac{\zeta(2 s) \zeta(3 s)}{\zeta(6 s)},
\end{aligned}
$$

for $\Re s>1 / 2$. Hence,

$$
\begin{aligned}
& M_{\text {square-full, } \alpha, \beta}(x) \\
& \quad=\frac{1}{2 \pi i} \int_{c-i \infty}^{c+i \infty} \frac{x^{s}}{s(s+1)} \cdot \frac{\zeta(2 s-2 \alpha) \zeta(3 s-3 \alpha) \zeta(2 s-2 \beta) \zeta(3 s-3 \beta)}{\zeta(6 s-6 \alpha) \zeta(6 s-6 \beta)} d s,
\end{aligned}
$$

for $c>1 / 2+\max (\alpha, \beta)$. For brevity's sake, let the integrand above be denoted by $V(s)$. For simplicity, we discuss in detail only the case $\alpha=\beta$. The cases $\alpha>\beta$ and $\alpha<\beta$ can be dealt with in a similar way.

Putting $\alpha=\beta$ in (3), we obtain

$$
M_{\text {square-full }, \alpha, \beta}(x)=\frac{1}{2 \pi i} \int_{c-i \infty}^{c+i \infty} \frac{x^{s}}{s(s+1)} \cdot \frac{\zeta^{2}(2 s-2 \alpha) \zeta^{2}(3 s-3 \alpha)}{\zeta^{2}(6 s-6 \alpha)} d s .
$$

We deform the line of integration into a path which consists of the union of nine line segments

$$
\begin{cases}s=\eta+i t, & \text { if }|t| \geq U, \\ s=\sigma \pm i U, & \text { if } \alpha+1 / 6 \leq \sigma \leq \eta, \\ s=\alpha+1 / 6+i t, & \text { if } T \leq|t| \leq U, \\ s=\sigma \pm i T, & \text { if } \nu \leq \sigma \leq \alpha+1 / 6, \\ s=\nu+i t, & \text { if }|t| \leq T,\end{cases}
$$


where $0<T<U$ are parameters to be chosen later. Let $J_{1}, \ldots, J_{9}$ be the line integrals on the corresponding line segments. Also, we set

$$
\eta=\alpha+\frac{1}{2}+\frac{c_{1}}{\log x}
$$

and

$$
\nu=\alpha+\frac{1}{6}-\frac{c_{2}}{(\log T)^{2 / 3}(\log \log T)^{1 / 3}},
$$

for some constants $c_{1}, c_{2}>0$. We will employ the Vinogradov-Korobov type zerofree region (see Titchmarsh [7], p. 135)

$$
\sigma \geq 1-\frac{c_{3}}{(\log t)^{2 / 3}(\log \log t)^{1 / 3}}, \quad t \geq t_{0}
$$

where $c_{3}>0$ is an absolute constant, in which

$$
\frac{1}{|\zeta(s)|}=O\left((\log t)^{2 / 3}(\log \log t)^{1 / 3}\right)
$$

Then, with an appropriate choice for $c_{2}$, the function $\zeta(6 s-6 \alpha)$ will have no zeros on or within the modified contour above. So the only poles of the integrand $V(s)$ inside the contour are double poles at $s=\alpha+1 / 2$ and $s=\alpha+1 / 3$. Letting the apostrophe symbol stand for the derivative with respect to $s$ and using $\gamma$ to denote the Euler constant, we see that the residue at $s=\alpha+1 / 2$ is

$$
\operatorname{Res}(V(s) ; \alpha+1 / 2)=A_{1}(\alpha+1 / 2) \gamma+\frac{1}{4} A_{1}^{\prime}(\alpha+1 / 2),
$$

where

$$
A_{1}(s)=\frac{x^{s}}{s(s+1)} \cdot \frac{\zeta^{2}(3 s-3 \alpha)}{\zeta^{2}(6 s-6 \alpha)} .
$$

Similarly, the residue at $s=\alpha+1 / 3$ is

$$
\operatorname{Res}(V(s) ; \alpha+1 / 3)=\frac{2}{3} A_{2}(\alpha+1 / 3) \gamma+\frac{1}{9} A_{2}^{\prime}(\alpha+1 / 3),
$$

where

$$
A_{2}(s)=\frac{x^{s}}{s(s+1)} \cdot \frac{\zeta^{2}(2 s-2 \alpha)}{\zeta^{2}(6 s-6 \alpha)} .
$$


After a short calculation, we find that

$$
\begin{aligned}
\operatorname{Res}(V(s) ; \alpha+1 / 2)= & \frac{4 \zeta^{2}(3 / 2) \gamma}{\left(4 \alpha^{2}+8 \alpha+3\right) \zeta^{2}(3)} x^{\alpha+1 / 2}+\frac{\zeta(3 / 2)}{\left(4 \alpha^{2}+8 \alpha+3\right)^{2} \zeta^{3}(3)} \\
& \times\left(\left(4 \alpha^{2}+8 \alpha+3\right) \zeta(3) \zeta(3 / 2) \log x-2\left\{-9 \zeta(3) \zeta^{\prime}(3 / 2)\right.\right. \\
& +2 \zeta(3 / 2)\left(2 \zeta(3)+9 \zeta^{\prime}(3)\right)-12 \alpha^{2}\left(\zeta(3) \zeta^{\prime}(3 / 2)-2 \zeta(3 / 2) \zeta^{\prime}(3)\right) \\
& \left.\left.+4 \alpha\left[-6 \zeta(3) \zeta^{\prime}(3 / 2)+\zeta(3 / 2)\left(\zeta(3)+12 \zeta^{\prime}(3)\right)\right]\right\}\right) x^{\alpha+1 / 2}
\end{aligned}
$$

and that

$$
\begin{aligned}
\operatorname{Res}(V(s) ; \alpha+1 / 3)= & \frac{216 \zeta^{2}(2 / 3) \gamma}{\pi^{4}\left(9 \alpha^{2}+15 \alpha+4\right)} x^{\alpha+1 / 3}+\frac{36 \zeta(2 / 3)}{\pi^{6}\left(9 \alpha^{2}+15 \alpha+4\right)^{2}} \\
& \times\left(\pi^{2}\left(9 \alpha^{2}+15 \alpha+4\right) \zeta(2 / 3) \log x\right. \\
& +\pi^{2}\left(-15 \zeta(2 / 3)-18 \alpha \zeta(2 / 3)+16 \zeta^{\prime}(2 / 3)+60 \alpha \zeta^{\prime}(2 / 3)\right. \\
& \left.\left.+36 \alpha^{2} \zeta^{\prime}(2 / 3)\right)-72\left(9 \alpha^{2}+15 \alpha+4\right) \zeta(2 / 3) \zeta^{\prime}(2)\right) x^{\alpha+1 / 3} .
\end{aligned}
$$

For the case $\alpha>\beta$, the integrand $V(s)$ has a simple pole at $s=\alpha+1 / 2$, with residue

$$
\operatorname{Res}(V(s) ; \alpha+1 / 2)=\frac{\zeta(2 \alpha-2 \beta+1) \zeta(3 \alpha-3 \beta+3 / 2) \zeta(3 / 2)}{2(\alpha+1 / 2)(\alpha+3 / 2) \zeta(6 \alpha-6 \beta+3) \zeta(3)} x^{\alpha+1 / 2} .
$$

This provides the main term in the asymptotic formula for $M_{\text {square-full }, \alpha, \beta}(x)$. The integrand $V(s)$ also has poles at $s=\alpha+1 / 3, s=\beta+1 / 2$, and $s=\beta+1 / 3$, which produce smaller order terms. To this end, we remark that in the particular case when $\alpha=\beta+1 / 6, s=\alpha+1 / 3$ and $s=\beta+1 / 2$ coincide, and the integrand $V(s)$ has a pole of order two at this point. Consequently, in this particular case, the corresponding asymptotic formula for $M_{\text {square-full, } \alpha, \beta}(x)$ will have a main term of size $x^{\alpha+1 / 2}$ followed by a second term of size $x^{\alpha+1 / 3} \log x$. By symmetry, the asymptotic formula for $M_{\text {square-full, } \alpha, \beta}(x)$ in the case $\alpha<\beta$ is the same as that in the case $\alpha>\beta$, by interchanging the roles of $\alpha$ and $\beta$.

Returning to the case $\alpha=\beta, M_{\text {square-full, } \alpha, \beta}(x)$ equals the contribution of the residues of $V(s)$ at $s=\alpha+1 / 2$ and $=\alpha+1 / 3$ computed above, plus an error term coming from the nine line integrals. In virtue of the well-known estimates

$$
|\zeta(\sigma+i t)|= \begin{cases}O\left(t^{(1-\sigma) / 2} \log t\right), & \text { if } 0 \leq \sigma \leq 1, \\ O(\log t), & \text { if } 1 \leq \sigma \leq 2, \\ O(1), & \text { if } \sigma \geq 2,\end{cases}
$$

we proceed to estimate the integral along our modified contour. 
On the line segments on which $s=\eta+i t,|t| \geq U$, we have $|\zeta(2 \eta-2 \alpha+i 2 t)|=$ $O(\log x),|\zeta(3 \eta-3 \alpha+i 3 t)|=O(1)$, and $1 /|\zeta(6 \eta-6 \alpha+i 6 t)|=O(1)$, so that

$$
\begin{aligned}
\left|J_{1}\right|,\left|J_{9}\right| & =O\left(\int_{U}^{\infty} \frac{\left|x^{\eta+i t}\right|\left|\zeta^{2}(2 \eta-2 \alpha+i 2 t)\right|\left|\zeta^{2}(3 \eta-3 \alpha+i 3 t)\right|}{|\eta+i t||\eta+1+i t|\left|\zeta^{2}(6 \eta-6 \alpha+i 6 t)\right|} d t\right) \\
& =O\left(x^{\alpha+1 / 2}(\log x)^{2} \int_{U}^{\infty} \frac{d t}{t^{2}}\right) \\
& =O\left(\frac{x^{\alpha+1 / 2}(\log x)^{2}}{U}\right) .
\end{aligned}
$$

On the line segments on which $s=\sigma \pm i U, \alpha+1 / 6 \leq \sigma \leq \eta$, we have

$$
\begin{aligned}
& |\zeta(2 \sigma-2 \alpha+i 2 U)|=O\left(U^{(1-2 \sigma+2 \alpha) / 2} \log U\right) \text { if } \alpha+1 / 6 \leq \sigma \leq \alpha+1 / 2, \\
& |\zeta(2 \sigma-2 \alpha+i 2 U)|=O(\log U) \text { if } \alpha+1 / 2 \leq \sigma \leq \eta, \\
& |\zeta(3 \sigma-3 \alpha+i 3 U)|=O\left(U^{(1-3 \sigma+3 \alpha) / 2} \log U\right) \text { if } \alpha+1 / 6 \leq \sigma \leq \alpha+1 / 3, \\
& |\zeta(3 \sigma-3 \alpha+i 3 U)|=O(\log U) \text { if } \alpha+1 / 3 \leq \sigma \leq \eta, \text { and } \\
& 1 /|\zeta(6 \sigma-6 \alpha+i 6 U)|=O(\log U),
\end{aligned}
$$

so that

$$
\begin{aligned}
\left|J_{2}\right|,\left|J_{8}\right| & =O\left(\int_{\alpha+1 / 6}^{\eta} \frac{\left|x^{\sigma+i U}\right|\left|\zeta^{2}(2 \sigma-2 \alpha+i 2 U)\right|\left|\zeta^{2}(3 \sigma-3 \alpha+i 3 U)\right|}{|\sigma+i U||\sigma+1+i U|\left|\zeta^{2}(6 \sigma-6 \alpha+i 6 U)\right|} d \sigma\right) \\
& =O\left(\frac { ( \operatorname { l o g } U ) ^ { 6 } } { U ^ { 2 } } \left[\int_{\alpha+1 / 6}^{\alpha+1 / 3} x^{\sigma} U^{2-5 \sigma+5 \alpha} d \sigma\right.\right. \\
& \left.\left.+\int_{\alpha+1 / 3}^{\alpha+1 / 2} x^{\sigma} U^{1-2 \sigma+2 \alpha} d \sigma+\int_{\alpha+1 / 2}^{\eta} x^{\sigma} d \sigma\right]\right) \\
= & O\left(\frac { ( \operatorname { l o g } U ) ^ { 6 } } { U ^ { 2 } } \left[\max \left(x^{\alpha+1 / 3} U^{1 / 3}, x^{\alpha+1 / 6} U^{7 / 6}\right)\right.\right. \\
\left.\left.\quad+\max \left(x^{\alpha+1 / 2}, x^{\alpha+1 / 3} U^{1 / 3}\right)+x^{\eta}\right]\right) & \\
= & O\left(\frac{(\log U)^{6}}{U^{2}} \max \left(x^{\alpha+1 / 2}, x^{\alpha+1 / 3} U^{1 / 3}, x^{\alpha+1 / 6} U^{7 / 6}\right)\right) .
\end{aligned}
$$

On the line segments on which $s=\alpha+1 / 6+i t, T \leq|t| \leq U$, we have

$$
\begin{aligned}
& |\zeta(1 / 3+i 2 t)|=O\left(t^{1 / 3} \log t\right)=O\left(t^{1 / 3} \log U\right) \text { and } \\
& 1 /|\zeta(1+i 6 t)|=O(\log t)=O(\log U),
\end{aligned}
$$


so that

$$
\begin{aligned}
\left|J_{3}\right|,\left|J_{7}\right| & =O\left(\int_{T}^{U} \frac{\left|x^{\alpha+1 / 6+i t}\right|\left|\zeta^{2}(1 / 3+i 2 t)\right|\left|\zeta^{2}(1 / 2+i 3 t)\right|}{|\alpha+1 / 6+i t||\alpha+7 / 6+i t|\left|\zeta^{2}(1+i 6 t)\right|} d t\right) \\
& =O\left(x^{\alpha+1 / 6}(\log U)^{4} \int_{T}^{U} \frac{\left|\zeta^{2}(1 / 2+i 3 t)\right|}{t^{4 / 3}} d t\right) .
\end{aligned}
$$

Next, we decompose the interval of integration into diadic intervals. On each such interval, we employ the mean value formula

$$
\int_{0}^{X}\left|\zeta^{2}(1 / 2+i t)\right| d t=X \log X+(2 \gamma-1-\log 2 \pi) X+O\left(X^{1 / 2+\epsilon}\right)
$$

(see Titchmarsh [7], Theorem 7.4, p. 143) to derive

$$
\begin{aligned}
\int_{T}^{U} \frac{\left|\zeta^{2}(1 / 2+i 3 t)\right|}{t^{4 / 3}} d t & \leq \sum_{T / 2 \leq 2^{k} \leq U} \int_{2^{k}}^{2^{k+1}} \frac{\left|\zeta^{2}(1 / 2+i 3 t)\right|}{t^{4 / 3}} d t \\
& \leq \sum_{T / 2 \leq 2^{k} \leq U} \frac{1}{2^{4 k / 3}} \int_{2^{k}}^{2^{k+1}}\left|\zeta^{2}(1 / 2+i 3 t)\right| d t \\
& =O\left(\sum_{T / 2 \leq 2^{k} \leq U} \frac{1}{2^{4 k / 3}} \cdot k 2^{k}\right) \\
& =O\left(\frac{\log T}{T^{1 / 3}}\right) .
\end{aligned}
$$

It follows that

$$
\left|J_{3}\right|,\left|J_{7}\right|=O\left(\frac{x^{\alpha+1 / 6}(\log U)^{4} \log T}{T^{1 / 3}}\right)
$$

On the line segments on which $s=\sigma \pm i T, \nu \leq \sigma \leq \alpha+1 / 6$, we have

$$
\begin{aligned}
& |\zeta(2 \sigma-2 \alpha+i 2 T)|=O\left(T^{(1-2 \sigma+2 \alpha) / 2} \log T\right), \\
& |\zeta(3 \sigma-3 \alpha+i 3 T)|=O\left(T^{(1-3 \sigma+3 \alpha) / 2} \log T\right), \text { and } \\
& 1 /|\zeta(6 \sigma-6 \alpha+i 6 T)|=O\left((\log T)^{2 / 3}(\log \log T)^{1 / 3}\right),
\end{aligned}
$$


so that

$$
\begin{aligned}
\left|J_{4}\right|,\left|J_{6}\right| & =O\left(\int_{\nu}^{\alpha+1 / 6} \frac{\left|x^{\sigma+i T}\right|\left|\zeta^{2}(2 \sigma-2 \alpha+i 2 T)\right|\left|\zeta^{2}(3 \sigma-3 \alpha+i 3 T)\right|}{|\sigma+i T||\sigma+1+i T|\left|\zeta^{2}(6 \sigma-6 \alpha+i 6 T)\right|} d \sigma\right) \\
& =O\left(T^{5 \alpha}(\log T)^{16 / 3}(\log \log T)^{2 / 3} \int_{\nu}^{\alpha+1 / 6}\left(\frac{x}{T^{5}}\right)^{\sigma} d \sigma\right) \\
& =O\left(T^{5 \alpha}(\log T)^{16 / 3}(\log \log T)^{2 / 3}\left(\frac{x}{T^{5}}\right)^{\alpha+1 / 6}\left(\alpha+\frac{1}{6}-\nu\right)\right) \\
& =O\left(\frac{x^{\alpha+1 / 6}(\log T)^{14 / 3}(\log \log T)^{1 / 3}}{T^{5 / 6}}\right) .
\end{aligned}
$$

Lastly, on the line segment on which $s=\nu+i t,|t| \leq T$, we have

$$
\begin{aligned}
|\zeta(2 \nu-2 \alpha+i 2 t)| & =O\left(|t|^{(1-2 \nu+2 \alpha) / 2} \log (2+|t|)\right) \\
& =|t|^{1 / 3+O\left((\log T)^{-2 / 3}\right)} \log (2+|t|) \text { and } \\
1 /|\zeta(6 \nu-6 \alpha+i 6 t)|= & O\left((\log (2+|t|))^{2 / 3}(\log \log (3+|t|))^{1 / 3}\right),
\end{aligned}
$$

so that

$$
\begin{aligned}
\left|J_{5}\right|=O\left(\int_{-T}^{T} \frac{\left|x^{\nu+i t}\right|\left|\zeta^{2}(2 \nu-2 \alpha+i 2 t)\right|\left|\zeta^{2}(3 \nu-3 \alpha+i 3 t)\right|}{|\nu+i t||\nu+1+i t|\left|\zeta^{2}(6 \nu-6 \alpha+i 6 t)\right|} d t\right) \\
=O\left(x^{\nu} \int_{-T}^{T}|t|^{2 / 3+O\left((\log T)^{-2 / 3}\right)}(\log (2+|t|))^{10 / 3}\right. \\
\left.\times \frac{(\log \log (3+|t|))^{2 / 3}\left|\zeta^{2}(3 \nu-3 \alpha+i 3 t)\right|}{1+t^{2}} d t\right) .
\end{aligned}
$$

The last integral above is $O(1)$, since $|\zeta(3 \nu-3 \alpha+i 3 t)|=O\left((1+|t|)^{\theta}\right)$ for some fixed $\theta<1 / 6$. This, in turn, follows from known upper bounds for $\zeta(s)$ on the critical line (see Titchmarsh [7], Theorem 5.18, p. 113) which go beyond the classical result of Weyl (where the exponent equals $1 / 6$ ), combined with the fact that $3 \nu-3 \alpha+i 3 t=$ $1 / 2+i 3 t+O\left(1 /(\log T)^{2 / 3}\right)$. We deduce that

$$
\left|J_{5}\right|=O\left(x^{\nu}\right)=O\left(x^{\alpha+1 / 6-c_{2}(\log T)^{-2 / 3}(\log \log T)^{-1 / 3}}\right) .
$$

Collecting all estimates and taking

$$
T=\exp \left\{(\log x)^{3 / 5}(\log \log x)^{-1 / 5}\right\}
$$


and $U=x$, we obtain

$$
\sum_{i=1}^{9}\left|J_{i}\right|=O_{\alpha}\left(x^{\alpha+1 / 6} \exp \left\{-c_{4}(\log x)^{3 / 5}(\log \log x)^{-1 / 5}\right\}\right),
$$

for some constant $c_{4}>0$. The asymptotic formula for $M_{\text {square-full, } \alpha, \alpha}(x)$ from the statement of Theorem 1 now follows by considering the contribution of the term of size $x^{\alpha+1 / 2} \log x$ in $\operatorname{Res}(V(s) ; \alpha+1 / 2)$.

For the second part of Theorem 1, one starts with the Dirichlet series

$$
G_{\alpha, \beta}(x)=\sum_{n=1}^{\infty} \frac{\sigma_{\text {square }, \alpha, \beta}(n)}{n^{s}},
$$

which converges for $\Re s>1 / 2+\max (\alpha, \beta)$, and rewrites it as

$$
\begin{aligned}
G_{\alpha, \beta}(x) & =\sum_{n=1}^{\infty} \frac{1}{n^{s}} \sum_{\substack{d_{1} d_{2}=n \\
d_{1} \text { square } \\
d_{2} \text { square-full }}}^{\infty} d_{1}^{\alpha} d_{2}^{\beta} \\
& =\sum_{\substack{d_{1}=1 \\
d_{1} \text { square } d_{2}}}^{\infty} \sum_{\substack{d_{2}=1 \\
\text { square-full }}}^{\infty} \frac{d_{1}^{\alpha} d_{2}^{\beta}}{\left(d_{1} d_{2}\right)^{s}} \\
= & \sum_{\substack{d_{1}=1 \\
d_{1}}}^{\infty} \frac{1}{d_{1}^{s-\alpha}} \sum_{\substack{d_{2}=1 \\
d_{2} \text { square-full }}}^{\infty} \frac{1}{d_{2}^{s-\beta}} \\
= & \zeta(2 s-2 \alpha) H(s-\beta) .
\end{aligned}
$$

An application of Perron's formula then gives

$$
M_{\text {square }, \alpha, \beta}(x)=\frac{1}{2 \pi i} \int_{c-i \infty}^{c+i \infty} \frac{x^{s}}{s(s+1)} \cdot \frac{\zeta(2 s-2 \alpha) \zeta(2 s-2 \beta) \zeta(3 s-3 \beta)}{\zeta(6 s-6 \beta)} d s .
$$

This integral is similar to the one from the first part of Theorem 1, and a similar argument to the one above completes the proof of the theorem. 


\section{Generalizations}

If one is interested in more accurate asymptotic formulas than the ones stated in Theorem 1, one can derive such formulas from the proof above. There are many cases to consider, according to which of the points $s=\alpha+1 / 2, s=\alpha+1 / 3$, $s=\beta+1 / 2$, and $s=\beta+1 / 3$, are within or outside the contour of integration, and according to whether those points which lie inside the contour are distinct or overlapping, producing simple or double poles.

As examples, we conclude the paper by gathering below the resulting asymptotic formulas in some of these cases. If $\alpha=\beta$, then

$$
\begin{aligned}
M_{\text {square-full }, \alpha, \alpha}(x)= & \frac{4 \zeta^{2}(3 / 2) \gamma}{\left(4 \alpha^{2}+8 \alpha+3\right) \zeta^{2}(3)} x^{\alpha+1 / 2}+\frac{\zeta(3 / 2)}{\left(4 \alpha^{2}+8 \alpha+3\right)^{2} \zeta^{3}(3)} \\
& \times\left(\left(4 \alpha^{2}+8 \alpha+3\right) \zeta(3) \zeta(3 / 2) \log x-2\left\{-9 \zeta(3) \zeta^{\prime}(3 / 2)\right.\right. \\
& +2 \zeta(3 / 2)\left(2 \zeta(3)+9 \zeta^{\prime}(3)\right)-12 \alpha^{2}\left(\zeta(3) \zeta^{\prime}(3 / 2)-2 \zeta(3 / 2) \zeta^{\prime}(3)\right) \\
& \left.\left.+4 \alpha\left[-6 \zeta(3) \zeta^{\prime}(3 / 2)+\zeta(3 / 2)\left(\zeta(3)+12 \zeta^{\prime}(3)\right)\right]\right\}\right) x^{\alpha+1 / 2} \\
& +\frac{216 \zeta(2 / 3)^{2} \gamma}{\pi^{4}\left(9 \alpha^{2}+15 \alpha+4\right)} x^{\alpha+1 / 3}+\frac{36 \zeta(2 / 3)}{\pi^{6}\left(9 \alpha^{2}+15 \alpha+4\right)^{2}} \\
& \times\left(\pi^{2}\left(9 \alpha^{2}+15 \alpha+4\right) \zeta(2 / 3) \log x\right. \\
& +\pi^{2}\left(-15 \zeta(2 / 3)-18 \alpha \zeta(2 / 3)+16 \zeta^{\prime}(2 / 3)+60 \alpha \zeta^{\prime}(2 / 3)\right. \\
& \left.\left.+36 \alpha^{2} \zeta^{\prime}(2 / 3)\right)-72\left(9 \alpha^{2}+15 \alpha+4\right) \zeta(2 / 3) \zeta^{\prime}(2)\right) x^{\alpha+1 / 3} \\
& +O_{\alpha}\left(x^{\alpha+1 / 6} \exp \left\{-c(\log x)^{3 / 5}(\log \log x)^{-1 / 5}\right\}\right)
\end{aligned}
$$

and

$$
\begin{aligned}
M_{\text {square }, \alpha, \alpha}(x)= & \frac{4 \zeta(3 / 2) \gamma}{\left(4 \alpha^{2}+8 \alpha+3\right) \zeta(3)} x^{\alpha+1 / 2}+\frac{1}{\left(4 \alpha^{2}+8 \alpha+3\right)^{2} \zeta^{2}(3)}(-8 \zeta(3 / 2) \zeta(3) \\
& +\left(4 \alpha^{2}+8 \alpha+3\right) \zeta(3 / 2) \zeta(3) \log x+9 \zeta(3) \zeta^{\prime}(3 / 2)-18 \zeta(3 / 2) \zeta^{\prime}(3) \\
& +12 \alpha^{2}\left(\zeta(3) \zeta^{\prime}(3 / 2)-2 \zeta(3 / 2) \zeta^{\prime}(3)\right)-8 \alpha\left[-3 \zeta(3) \zeta^{\prime}(3 / 2)\right. \\
& \left.\left.+\zeta(3 / 2)\left(\zeta(3)+6 \zeta^{\prime}(3)\right)\right]\right) x^{\alpha+1 / 2} \\
& +O_{\alpha}\left(x^{\alpha+1 / 6} \exp \left\{-c(\log x)^{3 / 5}(\log \log x)^{-1 / 5}\right\}\right) .
\end{aligned}
$$


If $\alpha>\beta$ and $\alpha \neq \beta+1 / 6$, then

$$
\begin{aligned}
M_{\text {square-full }, \alpha, \beta}(x)= & \frac{\zeta(2 \alpha-2 \beta+1) \zeta(3 \alpha-3 \beta+3 / 2) \zeta(3 / 2)}{2(\alpha+1 / 2)(\alpha+3 / 2) \zeta(6 \alpha-6 \beta+3) \zeta(3)} x^{\alpha+1 / 2} \\
& +\frac{\zeta(2 \alpha-2 \beta+2 / 3) \zeta(3 \alpha-3 \beta+1) \zeta(2 / 3)}{3(\alpha+1 / 3)(\alpha+4 / 3) \zeta(6 \alpha-6 \beta+2) \zeta(2)} x^{\alpha+1 / 3} \\
& +\frac{\zeta(2 \beta-2 \alpha+1) \zeta(3 \beta-3 \alpha+3 / 2) \zeta(3 / 2)}{2(\beta+1 / 2)(\beta+3 / 2) \zeta(6 \beta-6 \alpha+3) \zeta(3)} x^{\beta+1 / 2} \\
& +\frac{\zeta(2 \beta-2 \alpha+2 / 3) \zeta(3 \beta-3 \alpha+1) \zeta(2 / 3)}{3(\beta+1 / 3)(\beta+4 / 3) \zeta(6 \beta-6 \alpha+2) \zeta(2)} x^{\beta+1 / 3} \\
& +O_{\alpha, \beta}\left(x^{\alpha+1 / 6} \exp \left\{-c(\log x)^{3 / 5}(\log \log x)^{-1 / 5}\right\}\right)
\end{aligned}
$$

and

$$
\begin{aligned}
M_{\text {square }, \alpha, \beta}(x)= & \frac{\zeta(2 \alpha-2 \beta+1) \zeta(3 \alpha-3 \beta+3 / 2)}{2(\alpha+1 / 2)(\alpha+3 / 2)} x^{\alpha+1 / 2} \\
& +\frac{\zeta(2 \beta-2 \alpha+1) \zeta(3 / 2)}{2(\beta+1 / 2)(\beta+3 / 2) \zeta(3)} x^{\beta+1 / 2} \\
& +\frac{\zeta(2 \beta-2 \alpha+2 / 3) \zeta(2 / 3)}{3(\beta+1 / 3)(\beta+4 / 3) \zeta(2)} x^{\beta+1 / 3} \\
& +O_{\alpha, \beta}\left(x^{\alpha+1 / 6} \exp \left\{-c(\log x)^{3 / 5}(\log \log x)^{-1 / 5}\right\}\right) .
\end{aligned}
$$

\section{References}

[1] P. T. Bateman, Square-full integers (solution to problem 4459), Amer. Math. Monthly 61 (1954), 477-479.

[2] G. H. Hardy and E. M. Wright, An Introduction to the Theory of Numbers, 5th ed., Clarendon Press, Oxford, 1979.

[3] S. Ramanujan, Highly Composite Numbers, Proc. London Math. Soc. 14 (1915), 347-409.

[4] P. Erdős and G. Szekeres, Über die Anzahl der Abelschen Gruppen grgrbener Ordnung und über ein verwandtes Zahlentheoretisches Problem, Acta Sci. Math. (Szeged) 7 (1934) 95-102.

[5] A. Sklar, Square-full integers (solutions to problem 4459), Amer. Math. Monthly 60 (1953), 55 .

[6] E. C. Titchmarsh, The Theory of Functions, 2nd ed., Oxford University Press, London, 1939. 
[7] E. C. Titchmarsh, The theory of the Riemann zeta-function, 2nd ed. (D. R. Heath-Brown, ed.), The Claredon Press, Oxford Univ. Press, New York, 1986. 